\title{
Work place lactation support program: an avenue to benefit workplace settings, employed women and infants
}

\begin{abstract}
Employed women are one of those groups who often find it challenging to continue their breastfeeding practices with paid work; hence they often up into stress and roleconflict due to their inability to manage infant's feeding responsibilities along with employment. Considering the rising trend of women's labor force participation and gradual decline in breastfeeding prevalence among employed women, in this paper, workplace lactation support program is discussed as an avenue that can not only sustain breastfeeding practices of employed women and improve maternal and child health in a society but can also benefit workplace organizations by enhancing job satisfaction, decreasing absenteeism and lowering turnover rates among female employees.
\end{abstract}

Volume I Issue I - 2015

\author{
Shela Akbar Ali Hirani \\ Aga Khan University School of Nursing and Midwifery, Pakistan
}

Correspondence: Shela Akbar Ali Hirani,Aga Khan University School of Nursing and Midwifery, Stadium Road, P.O. Box 3500, Karachi, Pakistan, Tel 0092-02I-348654I4,

Email shela.hirani@aku.edu

Received: June 25, 2015 | Published: July 02, 2015

Keywords: women, lactation, infant, health, workplace, benefits, support

\section{Introduction}

Breastfeeding is a complete diet for the young children, as well as an important aspect of parenting. Considering crucial role of breastfeeding towards improvement of maternal and child health in a society, World Health Organization has recommended to protect, promote support breastfeeding practices of lactating mothers at diverse care setups. ${ }^{1}$ Unfortunately, most of the time the efforts to protect, promote and support breastfeeding practices of women is limited to the health care setups and very little attention is paid at nursing mothers' workplace settings where employed women often juggle with the dual responsibilities of managing breastfeeding with employment. Literature highlights that employed women are one of those groups who often find it challenging to sustain their breastfeeding practices while returning to workplace setting. ${ }^{2-6}$

In today's century, rising economic recession, inflation and urbanization has not only tremendously increased women's workforce participation but has also resulted in gradual decline in breastfeeding prevalence among employed women. ${ }^{3,4}$ To address the issue of suboptimal breastfeeding rates among employed women, worldwide a pressing need is viewed to have lactation support programs at employed women's workplace settings. The notion of workplace lactation support program has been described in literature as supportive measures at workplace environment that facilitate employed women to sustain their breastfeeding practices during working hours. ${ }^{4,7,8}$ The worksite lactation support program is usually a company sponsored employee assistance program designed for lactating women that ensures provision of official breastfeeding breaks, breast milk pumping facilities, breast milk storage facilities, child daycare facilities and privacy for employed women to breastfeed their child or express breast milk at the workplace. ${ }^{4,9,10}$ It is asserted that workplace lactation program aims to accommodate rights and needs of nursing mothers and breastfeeding babies not only by offering various logistic arrangements at workplace but also through accommodation of mother and baby-friendly supportive policies at the workplace. ${ }^{11,12}$ Workplace lactation support programs are reflective of the planned and collective efforts of a workplace to facilitate breastfeeding practices of employed women through specialized arrangements. ${ }^{13}$ Literature highlights that although the offered support in such programs varies from workplace to workplace, these programs serve as work-life conflict resolution strategy for many employed women. ${ }^{14}$ In the light of literature, in this paper it is argued that workplace lactation support program is an avenue that can offer tremendous benefits for the employers/ workplace settings, employed women and young children, hence, benefit the society at large.

\section{Benefits of workplace lactation support program for employers/workplace settings}

Workplace lactation support program offers several benefits to employers and workplace settings. ${ }^{15}$ Firstly, workplace lactation support program serves as an avenue to enhance job satisfaction among female employees, hence, enables employers to boost productivity of female employees and establish mutually beneficial association with them. ${ }^{15-17}$ Secondly, lactation support program serves as an employee retention strategy for the employers, as the facilities offered through such programs entitle female workers to manage the dual responsibility of continuing breastfeeding with employment. ${ }^{15}$ Thirdly, lactation support program can serve as cost-effective strategy for the employers by promoting retention among female employees; ${ }^{16}$ hence, it can save the cost of hiring and training new employees. Beside above benefits, the workplace lactation support program can enhance company's productivity by reducing absenteeism rates among female employees. ${ }^{15,16}$ Employed women who are not well supported by their employers to sustain their breastfeeding practices during working hours often choose to formula feed their children which increases sickness rates among their babies and concurrently increases absenteeism among female employees. ${ }^{15}$ Moreover, workplace lactation support program by offering mother and baby friendly interventions at the workplace positively contributes towards improving an organizational image, ${ }^{18}$ hence serves as pull factor for employees to join the organization that values and respects employees' comfort and work-life balance. 


\section{Benefits of workplace lactation support programfor employed women}

Workplace lactation support benefits employed women in various ways. Worksite lactation support programs facilitate employed women to choose breastfeeding for their infants which is the safest and costeffective option as compared to the artificial formula feed. ${ }^{15,19,20}$ By providing an opportunity to employed women either to express their feed or directly breastfeed their babies during working hours, these programs promote exclusivity and continuation of breastfeeding for longer duration..$^{21}$ Ultimately, employed women's financial cost on buying expensive formula milk, feeders, and sterilizing equipment for cleaning feeders is saved. This indicates that such programs could equally serve as economical and cost-saving for the employed women and their family.

Workplace lactation support program by promoting breastfeeding practices of women enables employed women to return to pre-pregnancy weight earlier and remain safe from the risks of developing breast/ovarian cancer and osteoporosis..$^{15}$ As workplace lactation support program empowers women in fulfilling financial needs of their family while satisfying nutritional needs of their infants; therefore, mothers' return to work for earning money or pursuing career becomes easier, especially in situations when they do not hold any child care support at home. Hence, these programs at one hand enhance personal life achievement among employed women, and on other hand minimize that risk of domestic life conflict among them. The nature of support offered in such programs i.e. privacy, breastfeeding break for breast milk expression or direct feeding and day care facilities etc. enables employed women to maintain maternal-child attachment, as well as provides emotional satisfaction to employed women by preventing the disequilibrium in their employment and child care responsibilities. Literature also states, "Providing women with the equipment needed to meet lactation goals while at work is a step toward reducing behavior-based conflict". ${ }^{14}$

\section{Benefit of workplace lactation support program for young children}

Workplace lactation support program also offers several health benefits for the young children of employed women. These programs by facilitating employed women to sustain natural source of feeding for their infants contribute towards better growth and nutritional status of young children, ${ }^{15}$ hence, save them from the negative effects of malnutrition related mortalities and morbidities. Moreover, through promoting, protecting and supporting breastfeeding, lactation support programs can be instrumental in promoting brain development, expanding mental capacities, and lowering the probability of chronic diseases like asthma, cancers, diabetes etc. among young babies of employed women. ${ }^{15}$ Several researches indicate that breastfed children have higher cognitive abilities, greater motor abilities, better language acquisition, and effective school performance in later life as compared to children who are not breastfed. ${ }^{22-25}$ The difference in cognitive development of breastfeeding and bottle feeding children could be well understood in the light of a meta-analysis that concludes, "... breastfeeding is associated with a 3.16-point higher score for cognitive development compared with formula feeding after adjustment for significant covariate". ${ }^{26}$ In addition, workplace lactation support program by promoting the breastfeeding practices of employed women enables their infants to avail the immunological benefits of breastfeeding. Consequently, infants who receive breastfeeding during the first year of their lives experience lesser sickness as compared to those babies who receive formula feeding. Several studies when compared the rates of childhood sickness and hospitalization among infants belonging to breastfeeding group versus infants belonging to formula feed group then found much reduced rates of illness and hospital admissions among infants belonging to breastfeeding group. ${ }^{27,28}$ This indicates that workplace lactation support program can play a pivotal role in reducing the illness rates by boosting immunity among infants of employed women.

\section{Conclusion}

Workplace lactation support program is an avenue that is equally beneficial for the workplace settings, employed women and young children. While accommodating breastfeeding supportive measures i.e. physical facilities, mother-baby supportive policies and flexibility at the workplace these programs can not only contribute effectively towards enhancing company's reputation and productivity of female employees but can also promote maternal and child health at the societal level due to short term and long terms benefits of breastfeeding. Considering tremendous benefits of these programs, it is recommended that workplace lactation support initiatives must be made part of all workplace settings.

\section{Acknowledgements}

None.

\section{Conflict of interest}

The author declares no conflict of interest.

\section{References}

1. World Health Organization. Infant and young child nutrition: Technical consultation on infant and young child feeding. WHO: Fifty-third World Health Assembly; 2000.

2. Atabay E, Moreno G, Nandi A, et al. Facilitating working mothers' ability to breastfeed: global trends in guaranteeing breastfeeding breaks at work, 1995-2014. J Hum Lact. 2014;31(1):81-88.

3. Hirani SA, Premji SS. Mother's employment and breastfeeding continuation: global and Pakistani perspectives from the literature. $N P C H N$. 2009;12(2):18-24.

4. Hirani SA, Karmaliani R. Evidence based workplace interventions to promote breastfeeding practices among Pakistani working mothers. Women Birth. 2013;26(1):10-16.

5. Iellamo A, Sobel H, Engelhardt K. Working mothers of the World health organization western pacific offices: lessons and experiences to protect, promote, and support breastfeeding. J Hum Lact. 2015;31(1):36-39.

6. Skafida V. Juggling work and motherhood: the impact of employment and maternity leave on breastfeeding duration: a survival analysis on Growing Up in Scotland data. Matern Child Health J. 2012;16(2):519527

7. Bettinelli ME. Breastfeeding policies and breastfeeding support programs in the mother's workplace. J Matern Fetal Neonatal Med. 2012;25(supl4):81-82.

8. Marinelli KA, Moren K, Taylor JS, Ademy of Breastfeeding Medicine Breastfeeding support for mothers in workplace employment or educational settings: Summary statement. Breastfeed Med. 2013;8(1):137142. 
9. Brodribb WE. Breastfeeding women in the paid workforce. Breastfeed Med. 2015;10(6).

10. Ortiz J, McGilligan K, Kelly P. Duration of breast milk expression among working mothers enrolled in an employer-sponsored lactation program. Pediatr Nurs. 20054;30(2):111-119.

11. Dodgson JE, Chee YO, Yap TS. Workplace breastfeeding support for hospital employees. J Adv Nurs. 2004;47(1):91-100.

12. Katcher AL, Lanese MG. Breast-Feeding by employed mothers: A reasonable accommodation in the workplace. Pediatrics. 1985;75(4):644 647.

13. Bonoan R. Breastfeeding support at the workplace. Washington Business Group on Health. Washington: 2000;2:1-8.

14. Cardenas RA, Major DA. Combining employment and breastfeeding: Utilizing a work-family conflict framework to understand obstacles and solutions. J Bus Psychol. 2005;20(1):31-51.

15. Mills SP. Workplace lactation programs: a critical element for breastfeeding mothers' success. AAOHN J. 2009;57(6):227-231.

16. Angeletti MA. Workplace lactation program: A nursing friendly initiative. J Health Hum Serv Adm. 2008;31(2):223-239.

17. Waite WM, Christakis D. Relationship of maternal perceptions of workplace breastfeeding support and job satisfaction. Breastfeed Med. 2015;10(4):222-227.

18. Seijts Gerard H. Milking the organization? The effect of breastfeeding accommodation on perceived fairness and organizational attractiveness. J Bus Ethics. 2002;40(1):1-13.

19. Balkam AJ, Cadwell K, Fein SB. Effect of components of a workplace lactation program on breastfeeding duration among employees of a public-sector employer. Matern Child Health J. 2011;15(5):677-683.
20. Tsai SY. Impact of a breastfeeding-friendly workplace on an employed mother's intention to continue breastfeeding after returning to work. Breastfeed Med. 2013;8(2):210-216.

21. Bai Y, Wunderlich SM. Lactation accommodation in the workplace and duration of exclusive breastfeeding. J Midwifery Womens Health. 2013;58(6):690-696.

22. Belfort MB, Rifas-Shiman SL, Kleinman KP, et al. Infant feeding and childhood cognition at ages 3 and 7 years: effects of breastfeeding duration and exclusivity. JAMA Pediatr. 2013;167(9):836-844.

23. Jedrychowski W, Perera F, Jankowski J, et al. Effect of exclusive breastfeeding on the development of children's cognitive function in the Krakow prospective birth cohort study. Eur J Pediatr. 2012;171(1):151158.

24. Oddy WH, Li J, Whitehouse AJ, et al. Breastfeeding duration and academic achievement at 10 years. Pediatrics. 2011;127(1):e137-145.

25. Quigley MA, Hockley C, Carson C, et al. Breastfeeding is associated with improved child cognitive development: a population-based cohort study. J Pediatr. 2012;160(1):25-32.

26. Anderson JW, Johnstone BM, Remley DT. Breast-feeding and cognitive development: a meta-analysis. Am J Clin Nutr. 1999;70(4):525-535.

27. Cohen R, Mrtek MB, Mrtek RG. Comparison of maternal absenteeism and infant illness rates among breast-feeding and formula-feeding women in two corporations. Am J Health Promot. 1995;10(2):148-153.

28. Ajetunmobi OM, Whyte $\mathrm{B}$, Chalmers $\mathrm{J}$, et al. Breastfeeding is associated with reduced childhood hospitalization: evidence from a Scottish Birth Cohort (1997-2009). J Pediatr. 2015;166(3):620-625. 